\title{
The effect of free and carrier-bound cortisol on equine neutrophil function
}

Melanie A. Fratto ${ }^{a}$, Kelsey A. Hart ${ }^{\mathrm{a}}$, Natalie A. Norton ${ }^{\mathrm{a}}$, Michelle H. Barton ${ }^{\mathrm{a}}$, Steeve Giguère ${ }^{\mathrm{a}}$, David J. Hurley ${ }^{\mathrm{b}}$

From the Departments of ${ }^{\mathrm{a}}$ Large Animal Medicine and ${ }^{\mathrm{b}}$ Population Health, 2200 College Station Road, University of Georgia College of Veterinary Medicine, Athens, GA, USA, 30602

$\begin{array}{ll}\text { Corresponding author: } & \text { Dr. Kelsey A. Hart } \\ \text { Postal Address: } & \text { Department of Large Animal Medicine } \\ & \text { 2200 College Station Road } \\ & \text { University of Georgia College of Veterinary Medicine } \\ & \text { Athens, GA, USA, 30602 } \\ & 706-542-6326 \\ \text { Phone: } & 706-357-0108 \\ \text { Fax: } & \text { khart4@uga.edu } \\ \text { Email: } & \end{array}$




\begin{abstract}
Cortisol is a key anti-inflammatory hormone that increases in bacterial sepsis and circulates predominantly bound to cortisol binding globulin (CBG). Only unbound cortisol was believed to be biologically active, but recent evidence suggests that CBG-bound cortisol also regulates inflammation. The objective of this study was to evaluate the effects of free and CBGbound cortisol on equine neutrophil function ex vivo. We hypothesized that CBG would enhance cortisol-mediated suppression of neutrophil pro-inflammatory responses. Neutrophils isolated from 8 foals and 6 adult horses were exposed to Staphylococcus aureus antigen (SAA) alone and with hydrocortisone $(\mathrm{HC}), \mathrm{CBG}$, or both $(\mathrm{CBG}+\mathrm{HC})$. Inflammatory cytokine (TNF- $\alpha$, IL-8) and reactive oxygen species (ROS) production were measured and compared among stimulants and between ages with linear mixed-effects models. $\mathrm{CBG}$ and $\mathrm{CBG}+\mathrm{HC}$ inhibited ROS production induced by SAA in both foal and horse neutrophils, maintaining it at levels comparable to baseline production $(\mathrm{P} \leq 0.060-0.907)$. TNF- $\alpha$ production was not significantly different among stimulants $(\mathrm{P}=0.284) . \mathrm{CBG}+\mathrm{HC}$ significantly $(\mathrm{P} \leq 0.016)$ increased $\mathrm{IL}-8$ production by neutrophils in response to SAA in both foals and adults, although the response of foals was significantly greater than that of adults $(\mathrm{P}<0.001)$. These findings suggest that $\mathrm{CBG}$ directly modulates equine neutrophil responses, but the effects are cytokine- and age-specific.
\end{abstract}

\title{
Keywords
}

Foal, horse, sepsis, adrenal insufficiency, steroid 


\section{Introduction}

Bacterial sepsis is a leading cause of mortality in foals (Brewer, 1988; Cohen, 1994;

Hoffman et al., 1992; Marsh and Palmer, 2001), and can lead to shock, multiple organ

dysfunction syndrome, and circulatory failure, particularly when the inflammatory response to the initial bacterial infection is excessive or unregulated (Monk et al., 2014). The endocrine system is an important regulator of this inflammatory response, primarily through stimulation of the hypothalamic-pituitary-adrenal (HPA) axis to ensure appropriate production of the antiinflammatory glucocorticoid hormone cortisol (Bailey, 2010; Hart et al., 2011; Marik, 2009). However, immature HPA axis function characterized by limited cortisol production occurs in healthy neonatal foals (Broughton Pipkin et al., 1984; Hart et al., 2009b; Ousey et al., 2004). Further, in up to $50 \%$ of septic foals, severe transient HPA axis dysfunction termed critical illness-related corticosteroid insufficiency (CIRCI) can develop, and is associated with increased disease severity and decreased survival (Gold et al., 2007; Hart et al., 2009a; Hurcombe et al., 2008; Wong et al., 2009) CIRCI may also occur in critically ill horses

Specific mechanisms resulting in the pathogenesis of CIRCI in sepsis are not fully understood. Both septic foals and horses with the systemic inflammatory response syndrome (SIRS) or sepsis can exhibit signs of cortisol insufficiency such as hypotension and dysregulated, exaggerated inflammatory responses despite increased serum cortisol concentrations compared to healthy age-matched animals (Gold et al., 2007; Hart et al., 2011; Hart et al., 2009a;

Hurcombe et al., 2008; Mair et al., 2014; Wong et al., 2009). Clinical signs of CIRCI in the face of high circulating cortisol might result from peripheral target tissue resistance to cortisol, as is evidenced in several studies in people and rodents (Cohen and Venkatesh, 2009; Hendrix et al., 2002; Liu et al., 1993; Marik, 2009; Meduri et al., 2005). Peripheral glucocorticoid resistance has 
recently been described in adult horses with SIRS and is associated with increased mortality in this population (Hoffman et al., 2015).

Such peripheral cortisol resistance could be due to an imbalance between free and protein-bound cortisol in sepsis. Cortisol is lipophilic and thus circulates in plasma predominantly bound ( $90 \%)$ to plasma proteins such as cortisol binding globulin (CBG) and albumin; however, because cortisol receptors are intracellular, the 5-10\% of cortisol that is free in the plasma is generally presumed to be the biologically active fraction (Hart et al., 2011). There is evidence, though, that CBG-bound cortisol plays a critical role in mediating local and systemic inflammation by delivering and releasing cortisol to areas of active inflammation (Bailey, 2010; Hammond et al., 1990). CBG-deficient mice - with 100\% free cortisol - showed exaggerated pro-inflammatory responses and increased mortality in an experimental sepsis model compared to wild-type mice with $\sim 90 \%$ bound cortisol (Petersen et al., 2006). Recent studies utilizing a newly validated ELISA assay for equine CBG have documented 25\% lower circulating CBG concentrations in healthy neonatal foals as compared to adult horses, and further decreases in plasma CBG concentration in septic foals as in septic people (Adcock et al., 2006; Beishuizen et al., 2001; Fratto et al., 2015; Molenaar et al., 2011; Nenke et al., 2015).

Given the key role for $\mathrm{CBG}$ in regulating neutrophil action in other species, and this recent work illustrating substantially decreased CBG availability in healthy and septic neonatal foals, CBG-deficiency could play a role in the poorly regulated inflammatory responses and immunologic immaturity in newborn foals. Further, it might contribute to the pathogenesis of CIRCI in septic foals. The objective of this study was to evaluate the effects of free and CBGbound cortisol on equine foal and adult horse neutrophil function using an ex vivo bacterial sepsis model. We hypothesized that cortisol-mediated suppression of neutrophil reactive oxygen 
species (ROS) production and inflammatory cytokine production would be enhanced in the presence of CBG.

\section{Materials and Methods}

\subsection{Animals}

8 healthy neonatal foals ( 3 males and 5 females) and 6 healthy adult horses ( 5 geldings and 1 mare) were used. Foal breeds included 6 Quarter Horses, 1 Paint, and 1 Saddlebred. All foals were sampled at 24-48 hours of age. Adult horses included 5 warmbloods and 1 Quarter Horse, with a mean age of $16.3 \pm 3.6$ years (range $13-22$ years). All animals were determined to be systemically healthy before sampling based on normal physical examination findings. Additionally, all foals were full term (> 330 days gestation) and had adequate transfer of passive immunity, with plasma immunoglobulin $\mathrm{G}$ concentration of $\geq 800 \mathrm{mg} / \mathrm{dl}$ documented prior to sampling. All mare/foal pairs and adult horses were maintained on pasture or in a stall with daily paddock turnout as per their normal routine for at least 24 hours prior to sample collection on their home farm. Both university owned-and client-owned animals were sampled. The methods used in this study were approved by both the University of Georgia's Animal Care and Use Committee and the University of Georgia College of Veterinary Medicine's Clinical Research Committee. Informed owner consent was obtained before enrollment of client-owned animals.

\subsection{Sample Collection}

$60 \mathrm{~mL}$ of whole blood was drawn from the jugular vein of each foal and horse into a syringe containing $2 \mathrm{~mL} 100 \mu \mathrm{M}$ EDTA while the animal was controlled with brief standing restraint. Foals were not removed from their dam for sample collection. Samples were maintained at ambient temperature until neutrophil isolation within 30-120 minutes. All blood 
samples from foals and adult horses were collected during the same season (spring/summer) over a four-month period.

\subsection{Neutrophil Reactive Oxygen Species (ROS) Production}

Peripheral blood neutrophils were isolated within 120 minutes of collection with densitygradient centrifugation over Ficoll-Paque (1.077 gm/mL, GE Healthcare, Uppsala, Sweden) as previously described (Hart et al., 2011). Cell viability and concentration were assessed using a $0.04 \%$ trypan blue solution, and cell viability was greater than $95 \%$ for all animals. Neutrophils were then suspended in complete media (RPMI 1640 without phenol red [Mediatech, Manasses, VA] $+10 \%$ heat-inactivated low endotoxin fetal bovine serum [Hyclone, Logan, UT] $+1 \%$ Lglutamine $+0.1 \%$ gentamicin) to a concentration of $3 \times 10^{6}$ cells $/ \mathrm{ml}$. Prior to the start of the study, CBG and cortisol were determined to be undetectable in the batch of fetal bovine serum used herein (data not shown). was Samples $(100 \mu \mathrm{L})$ were plated in 96 well flat bottom sterile plates.

Neutrophil ROS response to Staphylococcus aureus whole cell antigen [SAA; prepared in the UGA College of Veterinary Medicine Applied Immunology Laboratory as described previously (Nace et al., 2014; Ryman et al., 2013)] at a 1:500 dilution in media or to phorbol myristate acetate (PMA, $10^{-7} \mathrm{M}$; Molecular Probes, Eugene, OR) was measured using a previously described flurometric assay (Donovan et al., 2007). Specifically, this method utilizes a fluorescent plate reader to measure fluorescent-mediated dye reduction (dihydrorhodamine 123) to a fluorescent product to quantify both intra- and extracellular ROS, including nitric oxide and hydrogen peroxide.(Crow, 1997; Hempel et al., 1999; Henderson and Chappell, 1993; Royall and Ischiropoulos, 1993) Cells were exposed to SAA to assess toll-like receptor-induced ROS production, while measurement of ROS production in response to PMA allowed for 
assessment of overall ROS production capacity. This dilution of SAA was used for stimulation instead of endotoxin as described previously (Hart et al., 2011), because in preliminary studies it provided optimal neutrophil stimulation while maintaining cell viability $\geq 90 \%$ with less interhorse variability in ROS response than to endotoxin (data not shown). Cells were exposed to SAA or PMA alone (positive control) and in the presence of hydrocortisone (HC, $15 \mu \mathrm{g} / \mathrm{dL}$; Solu-Cortef, Upjohn, New York, NY), cortisol binding globulin (CBG, $100 \mu \mathrm{g} / \mathrm{mL}$;

MyBioSource, San Diego, CA), or both for 2 hours. SAA and all treatments were added to cells simultaneously. Cells exposed to media alone served as a negative control. These HC and CBG concentrations were chosen to represent physiologically relevant concentrations determined in previous studies of neonatal foals and horses during health and sepsis/SIRS (Fratto et al., 2015; Hart et al., 2011; Hart et al., 2009b). All samples were run in quadruplicate and averaged, except for those containing CBG, which were run in duplicate and averaged. Cell viability was not reevaluated after stimulation and $\mathrm{HC} / \mathrm{CBG}$ treatment in this assay.

\subsection{Neutrophil Pro-inflammatory Cytokine Production}

Neutrophils were isolated as above and suspended in complete media (RPMI 1640 without phenol red $+10 \%$ fetal bovine serum $+1 \%$ L- glutamine $+0.1 \%$ gentamicin; Mediatech, Manasses, VA) to a concentration of $1.1 \times 10^{7}$ cells $/ \mathrm{mL}$ and incubated for 30 minutes at $37{ }^{\circ} \mathrm{C}$ and $5 \% \mathrm{CO}_{2} .900 \mu \mathrm{L}$ aliquots of the neutrophil suspension were transferred to sterile $12 \mathrm{X} 75$ snap top polystyrene tubes for exposure to SAA alone (positive control) and in the presence of $\mathrm{HC}$, $\mathrm{CBG}$, or both for 6 hours at the concentrations listed above for the ROS assay. Cells exposed to media only served as a negative control. Again, this dilution of SAA, these concentrations of HC and $\mathrm{CBG}$, and this stimulation duration were determined in preliminary studies to provide optimal neutrophil stimulation while maintaining cell viability $\geq 90 \%$ (data not shown). 
After incubation at $37{ }^{\circ} \mathrm{C}$ and $5 \% \mathrm{CO}_{2}$ for 6 hours, sample tubes were centrifuged at $400 \mathrm{x}$ $\mathrm{G}$ for 5 minutes and supernatants collected and stored at $-80^{\circ} \mathrm{C}$ until measurement of interleukin 8 (IL-8) concentration via a commercial equine ELISA (Equine IL-8 Do-It-Yourself ELISA, Kingfisher Biotech, Inc., St. Paul, MN). Tumor necrosis factor $\alpha$ (TNF- $\alpha)$ concentration was also measured using a validated equine ELISA developed in our laboratory as described previously (Figueiredo et al., 2008).

\subsection{Data Analysis}

A priori sample size calculation using preliminary data assessing neutrophil ROS production and IL-8 concentration after stimulation with SAA indicated that 4-6 animals per group would be required to detect a $30 \%$ reduction in ROS or IL-8 in the presence of CBG as being statistically significant with a set at 0.05 and statistical power set at $80 \%$. We elected to enroll 6-8 animals per group to allow for potential variation in observed values from preliminary values used for calculations. Normality of the data was assessed based on examination of histograms and normal Q-Q plots of the residuals. Constant variance of the data was assessed by plotting residuals against predicted values. ROS and IL-8 data were analyzed using linear mixed effects modeling with animal modeled as a random effect and age (foal vs. adult) and type of stimulant modeled as fixed nominal effects. Two-way interactions were also included in the model. Model fit was assessed using Akaike information criterion values. When indicated, multiple pairwise comparisons or comparisons of each type of stimulus to baseline were done using the Holm-Sidak test. TNF- $\alpha$ production data was only available in 4 foals due to assay failure for some samples, so effect of stimulant as above on TNF- $\alpha$ production in foal cells was analyzed using a one-way repeated measures ANOVA and multiple pairwise comparisons were performed with Tukey's test when indicated. Analysis was performed with commercial 
statistical software (SPSS version 23, IBM Corporation, Pittsburgh, PA, USA; Prism Version 5.0, GraphPad Software, La Jolla, CA, USA). For all analyses, $P<0.05$ was considered significant.

\section{Results and Discussion}

\subsection{ROS production in horses and foals}

ROS production from foal and adult horse neutrophils after exposure to SAA or PMA in the presence or absence of CBG, HC, or both is shown in Figure 1. There was a significant interaction between age and stimulant $(\mathrm{P}<0.001)$. In both foals and adult horses, ROS production in response to SAA or PMA alone was significantly increased as compared to baseline in unstimulated cells $(\mathrm{P} \leq 0.004)$. Further, in both foal and adult horse neutrophils exposed to SAA or PMA in the presence of HC, ROS production remained significantly increased compared to baseline ( $\mathrm{P}<0.001$ in adults, $\mathrm{P} \leq 0.037$ in foals). In contrast, when both foal and adult horse neutrophils were exposed to SAA or PMA in the presence of CBG, ROS production was not significantly different from baseline levels, regardless of the presence or absence of concurrent $\mathrm{HC}$ exposure $(\mathrm{P}=0.084-0.995)$.

Baseline ROS production was not significantly different between horses and foals $(\mathrm{P}=$ 0.994), but ROS responses to both SAA and PMA alone or in the presence of HC were significantly lower in foals than adults $(\mathrm{P} \leq 0.004)$. However, when cells were exposed to SAA or PMA in the presence of $\mathrm{CBG}$ with or without $\mathrm{HC}$, differences in ROS production between horses and foals were not statistically significant $(\mathrm{P}=0.060-0.907)$, though the difference did approach statistical significance for PMA + CBG $(\mathrm{P}=0.060)$. 
These results support our hypothesis that CBG is important for modulation of ROS production in equine neutrophils. In fact, incubation with SAA in the presence of $\mathrm{HC}$ alone failed to suppress ROS responses in foal or adult horse neutrophils, which was unexpected. However, this finding is supported by a previous in vivo study in which systemic administration of HC did not result in suppression of TLR-mediated or overall ROS production in foal neutrophils (Hart et al., 2011). Data from the present study, in which HC failed to suppress ROS production in both isolated foal and adult horse neutrophils without concurrent availability of CBG, suggest that the similar lack of HC-mediated ROS production in foals receiving systemic HC might be explained by $\mathrm{CBG}$ deficiency in those foals, as has been recently described.(Fratto et al., 2015) In fact, CBG-mediated suppression of ROS production in the study herein occurred irrespective of concurrent exposure to $\mathrm{HC}$ and was comparable between horses and foals, suggesting availability of CBG at adult horse levels may be critical for modulation of equine neutrophil inflammatory responses and could work through alternative pathways independent of intracellular steroid receptors in horses as in other species (Bailey, 2010; Hammond et al., 1990; Petersen et al., 2006).

\subsection{S. aureus antigen-induced inflammatory cytokine production in horses and foals}

IL-8 production from foal and adult horse neutrophils after exposure to SAA in the presence and absence of CBG, HC, or both is shown in Figure 2. There was a significant interaction between age and stimulant $(\mathrm{P}<0.001)$. In foals, IL-8 production was significantly increased in cells incubated with SAA in the presence of CBG, with or without HC, as compared to baseline in unstimulated cells and to cells exposed to SAA alone or in the presence of $\mathrm{HC}$ (P < 0.001 for all comparisons). In contrast, in adult horses, IL-8 production was significantly increased from baseline in cells exposed to SAA in the presence of $\mathrm{CBG}$ and $\mathrm{HC}$ together $(\mathrm{P}=$ 
0.016) and approached a statistically significant increase from baseline in cells concurrently exposed to SAA and $\mathrm{CBG}(\mathrm{P}=0.068)$, but there were no other significant differences among treatment groups $(\mathrm{P}=0.112-0.811)$.

Between-age comparisons revealed significantly higher IL-8 production in foal cells than adult cells exposed to SAA in the presence of CBG with or without $\mathrm{HC}(\mathrm{P}<0.001)$. There were no significant differences in IL-8 production between horses and foals at baseline or in response to SAA alone or in the presence of $\mathrm{HC}(\mathrm{P}=0.645-0.976)$.

TNF- $\alpha$ production from foal neutrophils in response to SAA in the different experimental conditions is shown in Figure 3. There were no significant differences in TNF- $\alpha$ production from foal neutrophils among the different treatment conditions $(P=0.284)$.

These data do not fully support our hypothesis, as we expected both $\mathrm{HC}$ and CBG to decrease SAA-induced inflammatory cytokine production as has been shown previously in vivo and in vitro for other species (Giamarellos-Bourboulis et al., 2010; Hammond et al., 1990; Petersen et al., 2006). TNF- $\alpha$ responses in the present study appeared to trend towards this pattern (see Figure 3), but differences among stimulant conditions did not reach statistical significance for any comparisons. Unfortunately, one TNF- $\alpha$ ELISA plate failed and could not be repeated due to sample volume constraints, so TNF- $\alpha$ data were not available for adult horses and were only available for 4 foals. Thus, given the small number of animals with available data and the wide inter-horse variation in induced TNF- $\alpha$ concentrations, this analysis may have been underpowered to detect significant differences among treatments.

A very different pattern of response to stimulants was observed for IL-8 production in both foal and adult horse cells. A significant increase in IL-8 production from baseline was not observed for cells exposed to SAA alone or in combination with HC, likely due to the wide inter- 
animal variation in overall IL-8 response. Most unexpectedly, the only samples in which IL-8 production was significantly or close-to-significantly increased from baseline in both adults and foals cells were those cells exposed to SAA in the presence of CBG. It appears from these data that CBG may actually stimulate rather than suppress production of IL-8 - a neutrophil chemokine that attracts leukocytes to sites of active inflammation - as part of its role in regulating local inflammation, and may exert this effect independent of cortisol. Nuclear factor

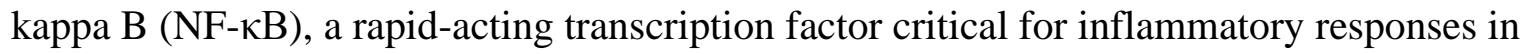
mammalian cells, is a key inducer of IL-8 production (Elliott et al., 2001; Takaki et al., 2003), and the cortisol-glucocorticoid receptor complex generally down regulates NF- $\kappa \mathrm{B}$ to decrease inflammatory cytokine production.(Meduri et al., 2005) Perhaps CBG, at least at the concentration and the exposure duration used in this study, alters or inhibits this regulation of $\mathrm{NF}-\kappa \mathrm{B}$ in equine neutrophils, resulting in increased rather than the expected decreases in IL-8 production. The degree of CBG-induced IL-8 production was also unexpectedly exaggerated in foals as compared to adult horses. This could be explained by up-regulated sensitivity to CBG in foal cells, given the normal state of relative CBG-deficiency in foal vs. adults as recently described.(Fratto et al., 2015)

In future studies, priming of the neutrophils with GM-CSF prior to bacterial stimulation as occurs in vivo - would be helpful to determine if the responses detected in the study herein are reproducible in more physiologically relevant circumstances. Further work investigating neutrophil exposure to $\mathrm{CBG}$ alone, in the absence of bacterial stimulation, is also needed to determine if $\mathrm{CBG}$, or components in the $\mathrm{CBG}$ preparation used in this study, have direct stimulatory or inhibitory effects on equine leukocytes. Finally, while little is known about mechanisms by which CBG may bind or signal through neutrophil or other cell membranes in 
any species,(Meyer and Rothuizen, 1993) future studies to quantify any potential impact of residual host CBG-cortisol-neutrophil interactions in similar ex vivo systems are needed.

\section{Conclusions}

In conclusion, $\mathrm{CBG}$ appears to be an important regulator of ROS production in equine neutrophils, perhaps working through an as-yet-undefined cell surface receptor(Nakhla et al., 1988; Strel'chyonok and Avvakumov, 1991) in a cortisol-independent fashion. CBG also appears to impact inflammatory cytokine production in a cortisol-independent manner, but effects vary among cytokines and between horses and foals. Thus, CBG should be considered as a contributing factor in the regulation of the innate immune response, rather than as a simple cortisol transporter. Further study is needed to determine signaling pathways through which CBG exerts these effects, and to evaluate the effects of varied ratios and timing of cortisol and CBG exposure on global measures of immune function to better reflect the diverse endocrine and immune response patterns seen during critical illness.

\section{Acknowledgements}

The authors would like to acknowledge Madi Berger for assistance with sampling and Loralei Branch for assistance with animal care. This study was supported by the USDA National Institute of Food and Agriculture, Animal Health Capacity Grant (Accession \#1004740), and a Morris Animal Foundation Veterinary Student Summer Research Award. Portions of these data were submitted for consideration for presentation as a Research Report at the Annual Forum of the American College of Veterinary Internal Medicine, Denver, CO, June 8-11, 2016. 


\section{References}

Adcock, R., Kattesh, H., Robert, M., Saxton, A., Carroll, J., 2006. Relationships between plasma cortisol, cortisosteroid-binding globulin $(\mathrm{CBG})$ and the free cortisol index (FCI) in pigs over a 24 hours period. J An Vet Adv 5, 85-91.

Bailey, S., 2010. Corticosteroid-associated laminitis. Vet Clin Equine 26, 277-285.

Beishuizen, A., Thijs, L., Vermes, I., 2001. Patterns of corticosteroid-binding globulin and the free cortisol index during septic shock and multitrauma. Intensive Care Med 27, 15841591.

Brewer, B., 1988. Neonatal foal evaluation: sepsis and survival scoring in private practice Thirty third Annual Conference of the American Association of Equine Practitioners. pp. 817822.

Broughton Pipkin, F., Ousey, J., Wallace, C., Rossdale, P., 1984. Studies on equine prematurity 4: Effect of salt and water loss on the renin-angiotensin-aldosterone system in the newborn foal. Equine Vet J 16, 292-297.

Cohen, J., Venkatesh, B., 2009. Assessment of tissue cortisol activity. Crit Care Resusc 11, 287289.

Cohen, N., 1994. Causes of and farm management factors associated with disease and death in foals. J Am Vet Med Assoc 204, 1644-1651.

Crow, J., 1997. Dichlorodihydrofluorescein and dihydrorhodamine 123 are sensitive indicators of peroxynitrite in vitro: implications for intracellular measurement of reactive nitrogen and oxygen species. Nitric Oxide: Biol Chem 1, 145-157. 
Donovan, D., Jackson, C., Colahan, P., Norton, N., Clapper, J., Hurley, D., 2007. Assessment of exercise-induced alterations in neutrophil function in horses. Am J Vet Res 68, 11981204.

Elliott, C.L., Allport, V.C., Loudon, J.A., Wu, G.D., Bennett, P.R., 2001. Nuclear factor-kappa B is essential for up-regulation of interleukin-8 expression in human amnion and cervical epithelial cells. Molecular human reproduction 7, 787-790.

Figueiredo, M., Moore, J., Vandenplas, M., Sun, W., Murray, T., 2008. Effects of the secondgeneration synthetic Lipid A analogue E5564 on responses to endotoxin in equine whole blood and monocytes. Am J Vet Res 69, 796-803.

Fratto, M., Hart, K., Norton, N., Berger, M., 2015. Cortisol binding globulin in septic foals, healthy foals and healthy horses (Research Abstract). J Vet Intern Med 29, 1241-1242.

Giamarellos-Bourboulis, E.J., Dimopoulou, I., Kotanidou, A., Livaditi, O., Pelekanou, A., Tsagarakis, S., Armaganidis, A., Orfanos, S.E., 2010. Ex-vivo effect of dexamethasone on cytokine production from whole blood of septic patients: correlation with disease severity. Cytokine 49, 89-94.

Gold, J., Divers, T., Barton, M., Lamb, S., Place, N., Mohammed, H., Bain, F., 2007. Plasma adrenocorticotropin, cortisol, and adrenocorticotropin/cortisol ratios in septic and normalterm foals. J Vet Intern Med 21, 791-796.

Hammond, G., Smith, C., Paterson, N., Sibbald, W., 1990. A role for corticosteroid-binding globulin in delivery of cortisol to activated neutrophils. J Clin Endocrin Metab 71, 34-39.

Hart, K., Barton, M., Ferguson, D., Berghaus, R., Slovis, N., Heusner, G., Hurley, D., 2011. Serum Free Cortisol Fraction in Healthy and Septic Neonatal Foals. Journal of Veterinary Internal Medicine 25, 345-355. 
Hart, K., Heusner, G., Norton, N., Barton, M., 2009a. Hypothalamic-pituitary-adrenal axis assessment in healthy term neonatal foals utilizing a paired low dose/high dose ACTH stimulation test. J Vet Intern Med 23, 344-351.

Hart, K., Slovis, N., Barton, M., 2009b. Hypothalamic-pituitary-adrenal axis dysfunction in hospitalized neonatal foals. J Vet Intern Med 23, 901-912.

Hempel, S., Buettner, G., O'Malley, Y., Wessels, D., Flaherty, D., 1999. Dihydrofluorescein diacetate is superior for detecting intracellular oxidants: comparison with 29,79Dichlorohydrofluorescein diacetate, 5(and 6)-carboxy-29,79-dichlorohydrofluorescein diacetate. and dihydrorhodamine 123. Free Radic Biol Med 27, 146-159.

Henderson, L., Chappell, J., 1993. Dihydrorhodamine 123: a fluorescent probe for superoxide generation. Eur J Biochem 217, 973-980.

Hendrix, D.V., Ward, D.A., Barnhill, M.A., 2002. Effects of anti-inflammatory drugs and preservatives on morphologic characteristics and migration of canine corneal epithelial cells in tissue culture. Vet Ophthalmol 5, 127-135.

Hoffman, A., Staempfli, H., Willan, A., 1992. Prognostic variables for survival of neonatal foals under intensive care. J Vet Intern Med 6, 89-95.

Hoffman, C.J., McKenzie, H.C., 3rd, Furr, M.O., Desrochers, A., 2015. Glucocorticoid receptor density and binding affinity in healthy horses and horses with systemic inflammatory response syndrome. J Vet Intern Med 29, 626-635.

Hurcombe, S., Toribio, R., Slovis, N., Kohn, C., Refsal, K., Saville, W., Mudge, M., 2008. Blood arginine vasopressin, adrenocorticotropin hormone, and cortisol concentrations at admission in septic and critically ill foals and their association with survival. J Vet Intern Med 22, 639-647. 
Liu, L., Sun, B., Tian, Y., Lu, B., Wang, J., 1993. Changes of pulmonary glucocorticoid receptor and phospholipase A2 in sheep with acute lung injury. Am Rev Respir Dis 148, 878-881.

Mair, T., Sherlock, C., Boden, L., 2014. Serum cortisol concentrations in horses with colic. Vet J 201, 370-377.

Marik, P., 2009. Critical illness-related corticosteroid insufficiency. Chest 135, 181-193.

Marsh, P., Palmer, J., 2001. Bacterial isolates from blood and their suspectibility patterns in critically ill foals: 543 cases (1991-1998). J Am Vet Med Assoc 218, 1608-1610.

Meduri, G., Muthiah, M., Carratu, P., Eltorky, M., Chrousos, G., 2005. Nuclear factor-kb- and glucocorticoid receptor-a mediated mechanisms in the regulation of systemic and pulmonary inflammation during sepsis and acute respiratory distress syndrome. Neuroimmunodulation 12, 321-338.

Meyer, H., Rothuizen, J., 1993. Determination of the percentage of free cortisol in plasma in the dog by ultrafiltration/dialysis. Dom An Endocrin 10, 45-53.

Molenaar, N., Johan Groeneveld, A., Dijstelbloem, H., de Jong, M., Girbes, A., Heijboer, A., Beishuizen, A., 2011. Assessing adrenal insufficiency of corticosteroid secretion using free versus total cortisol levels in critical illness. Intensive Care Med 37, 1986-1993.

Monk, C.S., Hart, K.A., Berghaus, R.D., Norton, N.A., Moore, P.A., Myrna, K.E., 2014. Detection of endogenous cortisol in equine tears and blood at rest and after simulated stress. Vet Ophthalmol 17 Suppl 1, 53-60.

Nace, E., Nickerson, S., Kautz, F., Briedling, S., Wochele, D., Ely, L., Hurley, D.J., 2014. Modualtion of innate immune function and phenotype in bred dairy heifers during the periparturient period induced by feeding an immunostimulant for 60 days prior to delivery. Vet Immunol Immunopathol 161, 240-250. 
Nakhla, A.M., Khan, M.S., Rosner, W., 1988. Induction of adenylate cyclase in a mammary carcinoma cell line by human corticosteroid-binding globulin. Biochem Biophys Res Commun 153, 1012-1018.

Nenke, M.A., Rankin, W., Chapman, M.J., Stevens, N.E., Diener, K.R., Hayball, J.D., Lewis, J.G., Torpy, D.J., 2015. Depletion of high-affinity corticosteroid-binding globulin corresponds to illness severity in sepsis and septic shock; clinical implications. Clinical endocrinology 82, 801-807.

Ousey, J., Rossdale, P., Fowden, A., Palmer, L., Turnbull, C., Allen, W., 2004. Effects of manipulating intrauterine growth on post natal adrenocortical development and other parameters of maturity in neonatal foals. Equine Vet J 36, 616-621.

Petersen, H., Andreassen, T., Breiderhoff, T., Brasen, J., Schulz, H., Gross, V., Grone, H., Nykjaer, A., Willnow, T., 2006. Hyporesponsiveness to glucocorticoids in mice genetically deficient for the corticosteroid binding globulin. Mol Cell Biol 26, 73267245.

Royall, J., Ischiropoulos, H., 1993. Evaluation of 2',7'-dichlorofluorescein and dihydrorhodamine-123 as fluorescent probes for intracellular $\mathrm{H} 2 \mathrm{O} 2$ in cultured endothelial cells. Arch Biochem Biophys 302, 348-355.

Ryman, V., Nickerson, S., Hurley, D., Berghaus, R., Kautz, F., 2013. Influence of horn flies (Haemotobia irritans) on teat skin condition, intramammary infection, and serum anti-S. aureus antibody titres in holstein heifers. Res Vet Sci 95, 343-346.

Strel'chyonok, O., Avvakumov, G., 1991. Interaction of human CBG with cell membranes. J Steroid Biochem Mol Biol 40, 795-803. 
Takaki, M., Ushikai, M., Deguchi, K., Nishimoto, K., Matsune, S., Kurono, Y., 2003. The role of nuclear factor-kappa B in interleukin-8 expression by human adenoidal fibroblasts. The Laryngoscope $113,1378-1385$.

Wong, D., Vo, D., Alcott, C., Peterson, A., Sponseller, B., Hsu, W., 2009. Baseline plasma cortisol and ACTH concentrations and response to low dose ACTH stimulation testing in ill foals. J Am Vet Med Assoc 234, 126-132.

\section{Figure Captions}


Figure 1. Mean \pm standard deviation reactive oxygen species (ROS) production measured in arbitrary fluorescent units (AFUs) in isolated foal $(\mathrm{n}=8)$ and adult horse $(\mathrm{n}=6)$ neutrophils after 2 hours of incubation with S. aureus antigen (SAA) or phorbol myristate acetate (PMA) in the presence or absence of hydrocortisone (HC), cortisol binding globulin (CBG), or both ( $\mathrm{HC}+$ $\mathrm{CBG})$. *Denotes significant $(\mathrm{P}<0.05)$ differences between horses and foals within a specific stimulus treatment. "Denotes significant $(\mathrm{P}<0.05)$ differences from baseline ROS production within each age group.

Figure 2. Mean \pm standard deviation interleukin- 8 (IL-8) production in isolated foal $(\mathrm{n}=8)$ and adult horse $(\mathrm{n}=6)$ neutrophils after 6 hours of incubation with S. aureus antigen (SAA) in the presence or absence of hydrocortisone (HC), cortisol binding globulin (CBG), or both (HC + $\mathrm{CBG})$. *Denotes significant $(\mathrm{P}<0.05)$ differences between horses and foals within a specific stimulus treatment. "Denotes significant difference $(\mathrm{P}<0.05)$ from baseline IL-8 production within each age group.

Figure 3. Mean \pm standard deviation tumor necrosis factor- $\alpha(\mathrm{TNF}-\alpha)$ production in isolated foal $(\mathrm{n}=8)$ neutrophils after 6 hours of incubation with S. aureus antigen (SAA) in the presence or absence of hydrocortisone $(\mathrm{HC})$, cortisol binding globulin $(\mathrm{CBG})$, or both $(\mathrm{HC}+\mathrm{CBG})$. 
$\square$ Foals

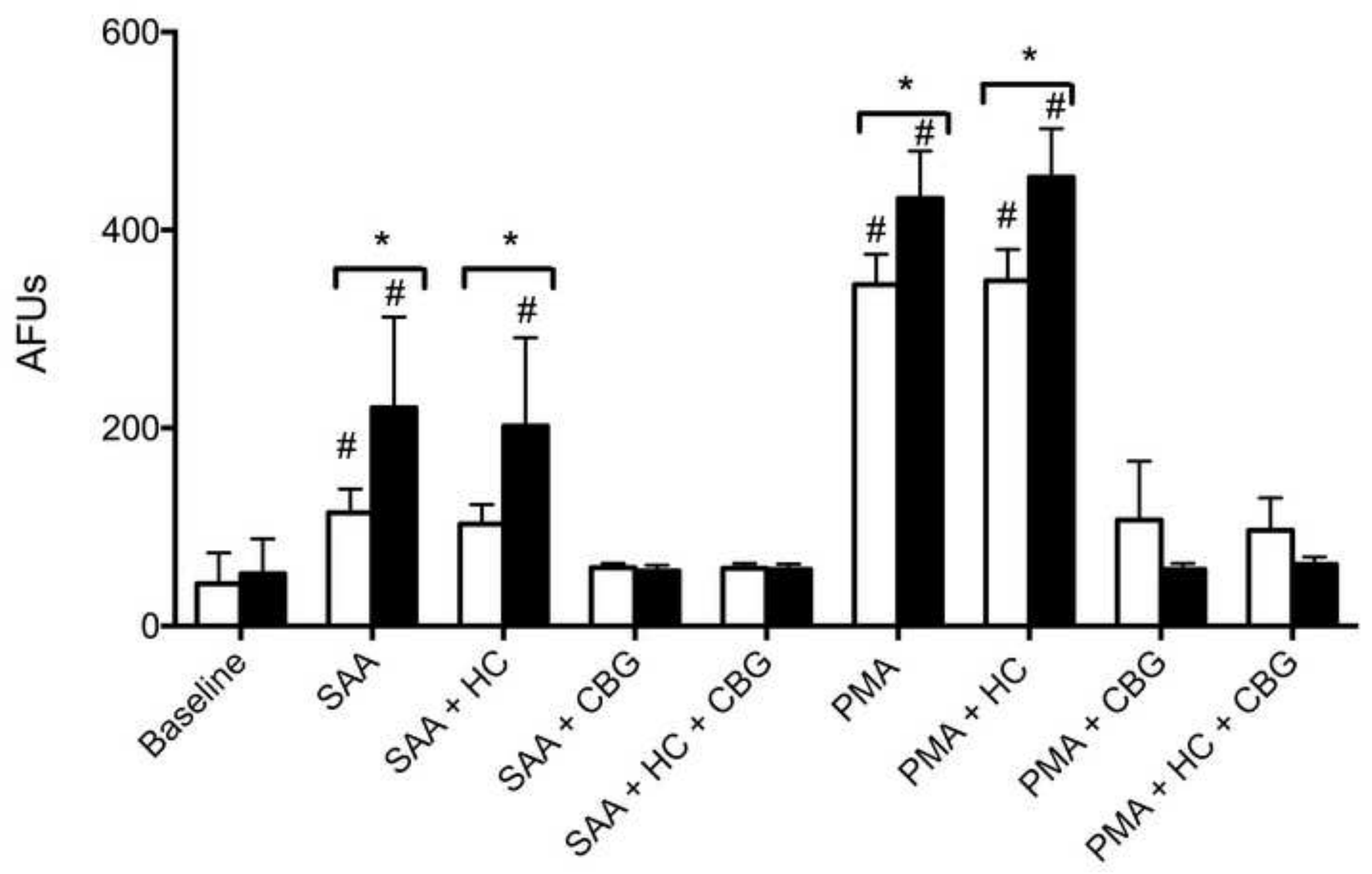


$\square$ Foels

Horees

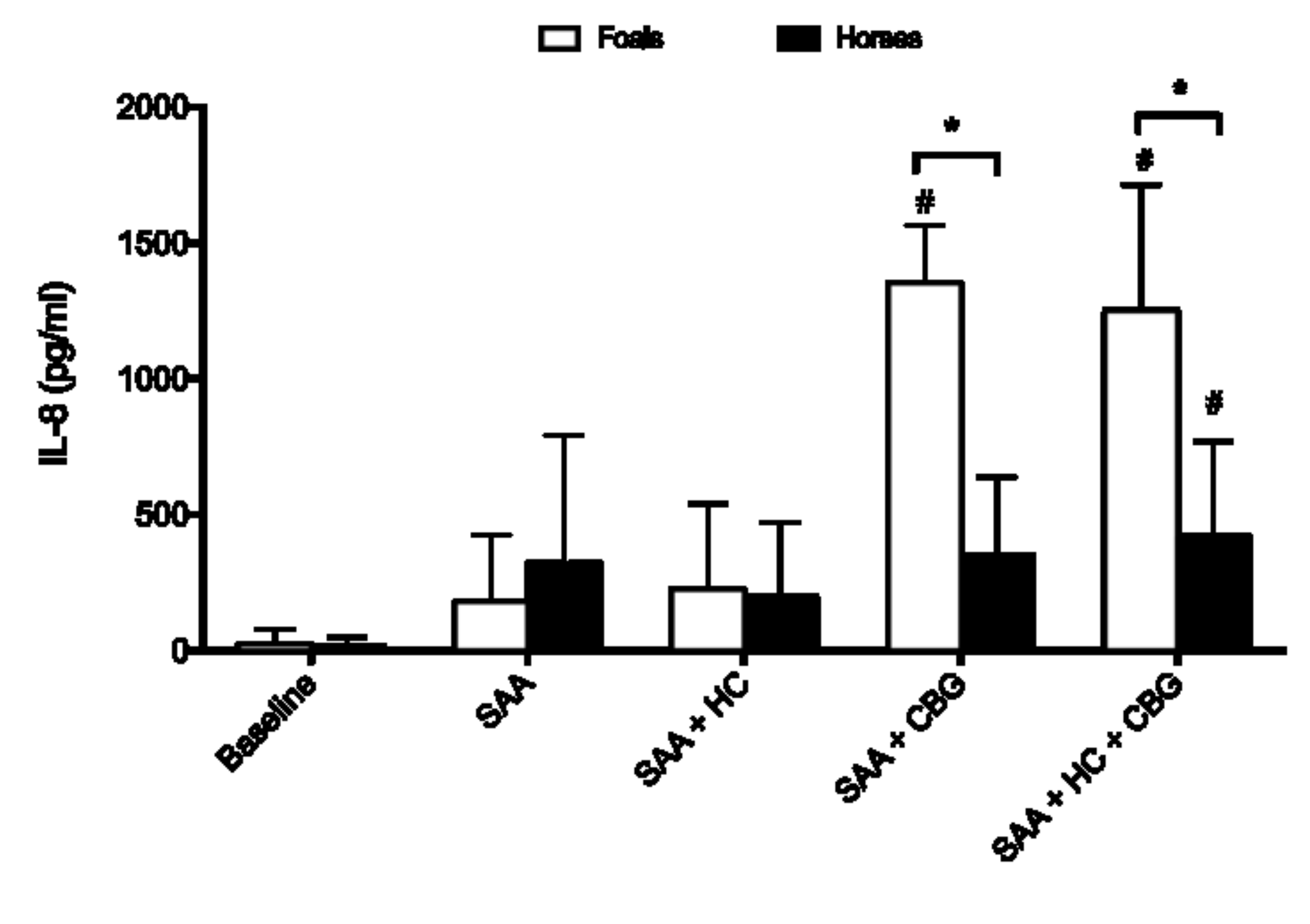

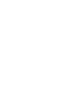
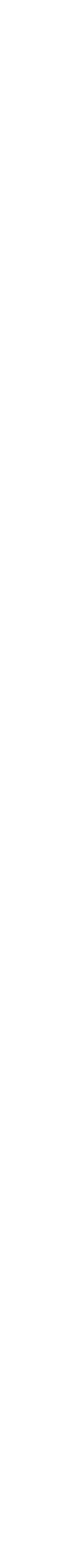


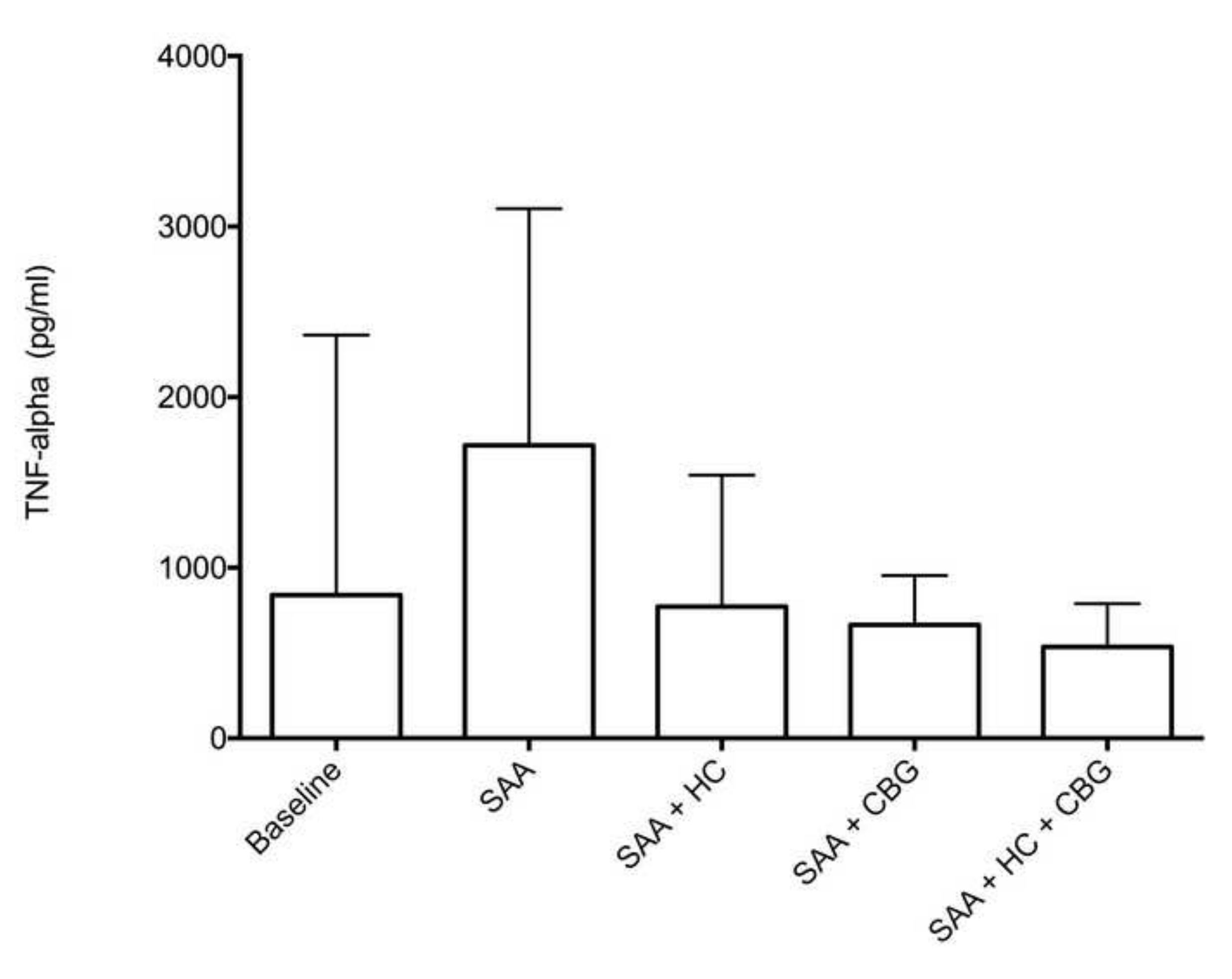

$$
\text { . }
$$

